\title{
Discovery research and translation science of trastuzumab deruxtecan, from non-clinical study to clinical trial
}

\author{
Takashi NAKADA ${ }^{1 *}$ \\ Oncology Research Laboratories I, Research Function, R\&D Division, Daiichi Sankyo Co., Ltd., 1-2-58 Hiromachi, Shinagawa-ku, Tokyo 140-8710, \\ Japan
}

\begin{abstract}
An antibody-drug conjugate (ADC) is a biological drug that binds a drug to a monoclonal antibody via an appropriate linker. ADCs use antibodies to selectively deliver a potent cytotoxic agent to tumor cells, thus drastically improving the therapeutic index of chemotherapeutic agents. We discovered trastuzumab deruxtecan (T-DXd) via study of ADC linker-payload technology that combined a DNA topoisomerase I inhibitor with an anti- human epidermal growth factor receptor 2 (HER2) antibody. T-DXd achieves a high drug-to-antibody ratio with homogeneous conjugation and is highly potent against heterogeneous tumors via the bystander antitumor effect. It is also considered to mitigate safety concerns in systemic circulation due to the linker-payload stability. The non-clinical profile of T-DXd was assessed and its pharmacological advantages were evaluated by in vivo xenograft studies. T-DXd was confirmed to be a valuable therapeutic tool with strong potential to treat breast cancer and other HER2-expressing cancers in a clinical setting. Indeed, T-DXd was recently approved for the treatment of patients with HER2-positive unresectable or recurrent breast cancers in the US, Japan, EU, UK, and Canada and those with HER2-positive unresectable or recurrent gastric cancers in the US and Japan.

Key words: trastuzumab deruxtecan, antibody-drug conjugate, cancer therapy, HER2, DNA topoisomerase I inhibitor
\end{abstract}

\section{Highlights}

1. The key features of trastuzumab deruxtecan (T-DXd) are its high potency of payload, high drug loading, linkerpayload stability, tumor-selectively cleavable, and bystander antitumor effect.

2. The non-clinical profile of T-DXd indicated its pharmacological advantages in xenograft mouse models with a wide range of HER2 expression levels and in a xenograft mouse model grafted with heterogeneous tumor tissues.

3. Safety, tolerability, pharmacokinetic profile, and efficacy of T-DXd were assessed in a clinical trial. In the DESTINYBreast 01 study, overall response rate of $60.9 \%$ and median progression-free survival of 16.4 months were achieved (cut-off date: August 1, 2019).

\section{Introduction}

An antibody-drug conjugate (ADC) provides an ideal drug delivery system (DDS) for cancer therapy because small-molecule agents are delivered to the targeted cancer cells to promote specific cancer cell killing [1, 2]. The essential components of ADCs are a potent cytotoxic drug, a highly specific monoclonal antibody, an appropriate linker connecting them, and a conjugation site related to drug distribution. 1) In terms of cytotoxicity, the drug requires a potent antitumor effect (in vitro cytotoxicity exerted in the sub-nanomolar range as reported previously [3]), since only a limited amount of drug can be delivered by an antibody. 2) The antibody also needs to target a highly expressed cancer

*Correspondence to: Nakada, T.:

nakada.takashi.g6@daiichisankyo.co.jp

Received: May 27, 2021; Accepted: Jul. 12, 2021 antigen for efficient delivery and to be internalized in the targeted cancer cells. 3) Regarding linker structure, it can be cleavable or non-cleavable. A cleavable linker needs to be cleaved rapidly in tumor cells but stable in circulation. The released payload containing the linker from a non-cleavable ADC needs to retain the efficacy of the original drug. 4) The optimal technology for conjugating the antibody to the linker-drug should be selected considering the appropriate number of drug molecules per antibody and distribution of the drug. Currently, site-specific conjugation technology is used as a solution for the complex distribution of drugs $[4,5]$.

In a clinical setting, the ADC approach increases the maximum therapeutic effect in cancer therapy and decreases undesirable side effects. Many breakthroughs and time-consuming research have been required to overcome various obstacles impeding the use of ADCs in a clinical setting $[6,7]$. As a result of numerous recent innovations, 
ten ADCs have to date been approved for cancer therapy by the US Food and Drug Administration (FDA) [8]: gemtuzumab ozogamicin, inotuzumab ozogamicin, trastuzumab emtansine, brentuximab vedotin, polatuzumab vedotin, enfortumab vedotin, trastuzumab deruxtecan, belantamab mafodotin, sacituzumab govitecan, and loncastuximab tesirine.

This article deals with trastuzumab deruxtecan (T-DXd) and describes the characteristic features of DXd-based ADC technology, the pharmacological effects, and pivotal clinical studies of T-DXd. T-DXd achieves a high drug-to-antibody ratio (DAR) with homogeneous conjugation, has strong potency against heterogeneous tumors via the bystander antitumor effect, and alleviates safety concerns related to its time in systemic circulation via high linker-drug stability. T-DXd could be a valuable therapeutic tool for effectively treating human epidermal growth factor receptor 2 (HER2)expressing cancers. Indeed, T-DXd was recently approved for the treatment of patients with HER2-positive unresectable or recurrent breast cancers in the US, Japan, and EU, and those with HER2-positive unresectable or recurrent gastric cancers in the US and Japan.

\section{Key Attributes of Trastuzumab Deruxtecan}

T-DXd is a HER2-targeting ADC for HER2-expressing solid tumors. It is prepared through the conjugation of a humanized anti-HER2 antibody with a novel DNA topoisomerase I (TOP1) inhibitor by using an enzymatically cleavable peptide-based linker, as shown in Fig. 1 [9].

The potency of DXd (payload of T-DXd) in inhibiting TOP1 was reported to be 10 -fold higher than that of SN-38, which is the active metabolite of irinotecan used for cancer therapy [10]. T-DXd achieved a high DAR of approximately 8 with site-specific and homogeneous conjugation on interchain cysteines prepared from the complete reduction of four interchain disulfide bonds of immunoglobulin G1 (IgG1). The DAR of 8 is theoretically the maximum drug loading number for conventional interchain cysteine conjugation. Despite this high DAR, the pharmacokinetic profile of T-DXd in cynomolgus monkeys was favorable. When T-DXd was intravenously administered at $3 \mathrm{mg} / \mathrm{kg}$, there was less difference between the ADC and the total antibody (both ADC and antibody with cleaved payload) in terms of plasma concentration compared with the other approved ADCs, as shown in Fig. 2A [10]. In addition, DXd was

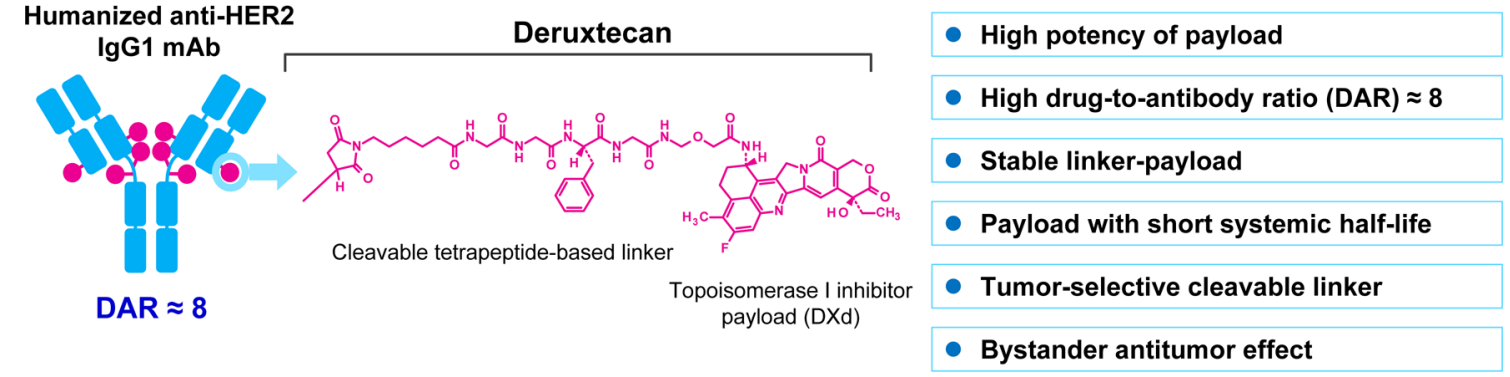

Fig. 1. Structure and seven attributes of trastuzumab deruxtecan.

A

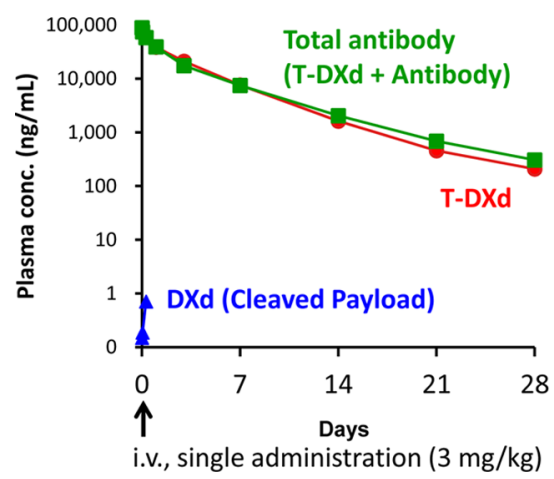

B

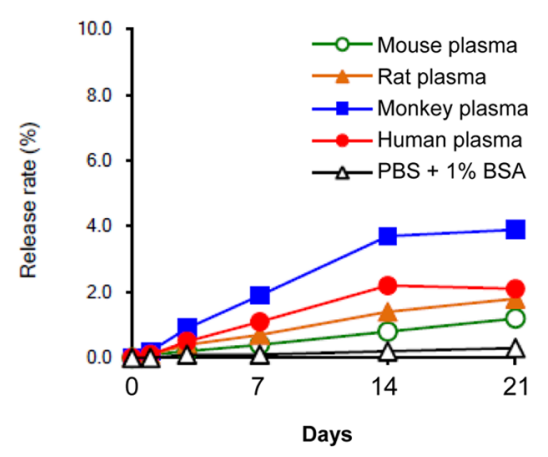

Fig. 2. Non-clinical study of trastuzumab deruxtecan (T-DXd) stability. A: Pharmacokinetics of T-DXd in cynomolgus monkeys. Mean plasma concentrations of T-DXd, total antibody, and DXd after the single intravenous administration of T-DXd at $3 \mathrm{mg} / \mathrm{kg}$ to cynomolgus monkeys. B: in vitro plasma stability of T-DXd in several species. PBS, phosphate Buffered Saline; BSA, bovine serum albumin. 
detected only at very early time points and at a much lower concentration than that of T-DXd; moreover, no linker-drug analog except DXd was detected in plasma [11, 12]. These results indicated that the linker-drug of T-DXd was stable in systemic circulation in cynomolgus monkeys. In addition, in vitro studies of stability in plasma were performed in several species, as shown in Fig. 2B. T-DXd showed high stability in plasma of all species, including cynomolgus monkey and human. In particular, the amount of DXd released from T-DXd in human plasma was also as low as $2.1 \%$ after 21 days of incubation [10].

The linker of T-DXd contains an enzymatically cleavable peptide (glycine-glycine-L-phenylalanine-glycine: GGFG [13]) and an amino-methylene moiety, which reduce hydrophobicity and provide stability in systemic circulation. The plausible mechanism of payload release is shown in Fig. 3 [9]. 1) The internalized ADC is selectively cleaved to a temporary hydrolysate composed of amino-methylene-DXd by lysosomal proteases such as cathepsins, which are known to be expressed in tumor cells. 2) The amino-methylene part is rapidly hydrolyzed to ammonia and formaldehyde. 3) The intracellular release of DXd occurs to trigger cell death.

DXd was designed to have cell membrane permeability in order to exert a bystander antitumor effect [14]. This effect could provide efficient killing of heterogeneous tumor cells, conferring a great advantage to T-DXd applied with this linker-payload system.

When DXd was intravenously administered at $1 \mathrm{mg} /$ $\mathrm{kg}$ in cynomolgus monkeys, the pharmacokinetic profile showed clearance and half-life of 2,390 $\pm 499 \mathrm{ml} / \mathrm{hr} / \mathrm{kg}$ and $1.37 \pm 0.766 \mathrm{hr}$, respectively [12]. From these results, such as rapid disappearance of payload from plasma, the exposure of normal tissues to DXd was expected to be low, given DXd's short half-life in systemic circulation and high linker-drug stability.

\section{Non-clinical Pharmacological Study of Trastuzumab Deruxtecan}

HER2 is a member of the epidermal growth factor receptor family of transmembrane receptors and is overexpressed in a broad range of cancer types, such as breast, gastric, colorectal, lung, and ovarian cancers [15, 16]. HER2 positivity with the overexpression of HER2 [immunohistochemistry (IHC) 3+ or IHC2+/in situ hybridization (ISH)+] was observed in $10 \%$ to $20 \%$ of breast cancer cases [17]. In addition, low expression of HER2 (IHC2+/ISH- or IHC1+) was reported to be observed in approximately $50 \%$ of breast cancer cases [18]. Trastuzumab emtansine (T-DM1), an ADC approved for HER2-positive cancer therapy, is known to show antitumor efficacy against HER2-positive breast cancer, but not against tumors with low HER2 expression [18].

The efficacy of T-DXd (DAR $\approx 8$ ) was compared with that of T-DM1 (DAR 3.5) and a lower-DAR HER2 ADC (DAR 3.4, using the same linker-payload as in T-DXd) in mouse xenograft models with different levels of HER2 expression, as shown in Fig. 4 [10]. T-DM1 showed efficacy only in KPL-4, a breast cancer cell line with high HER2 expression (meeting the clinical definition of HER2-positive by IHC). In contrast, T-DXd was effective in the cell line Capan-1, a pancreatic cell line with low HER2 expression, in addition to showing efficacy in KPL-4 and JIMT-1 (a T-DM1refractory HER2-positive breast cancer cell line). Neither ADC was effective in the GCIY cell line, a gastric cancer cell line negative for HER2 expression. The ADC with DAR of 3.4, applying the same linker-drug as T-DXd, exhibited antitumor activity against all levels of HER2 expression, but the degree was dependent on the expression of HER2; the activity of the ADC with DAR of 3.4 in Capan-1 cells with low HER2 expression was less than that of T-DXd with DAR of approximately 8 . These results show that the high DAR of T-DXd can deliver more payload to tumor cells

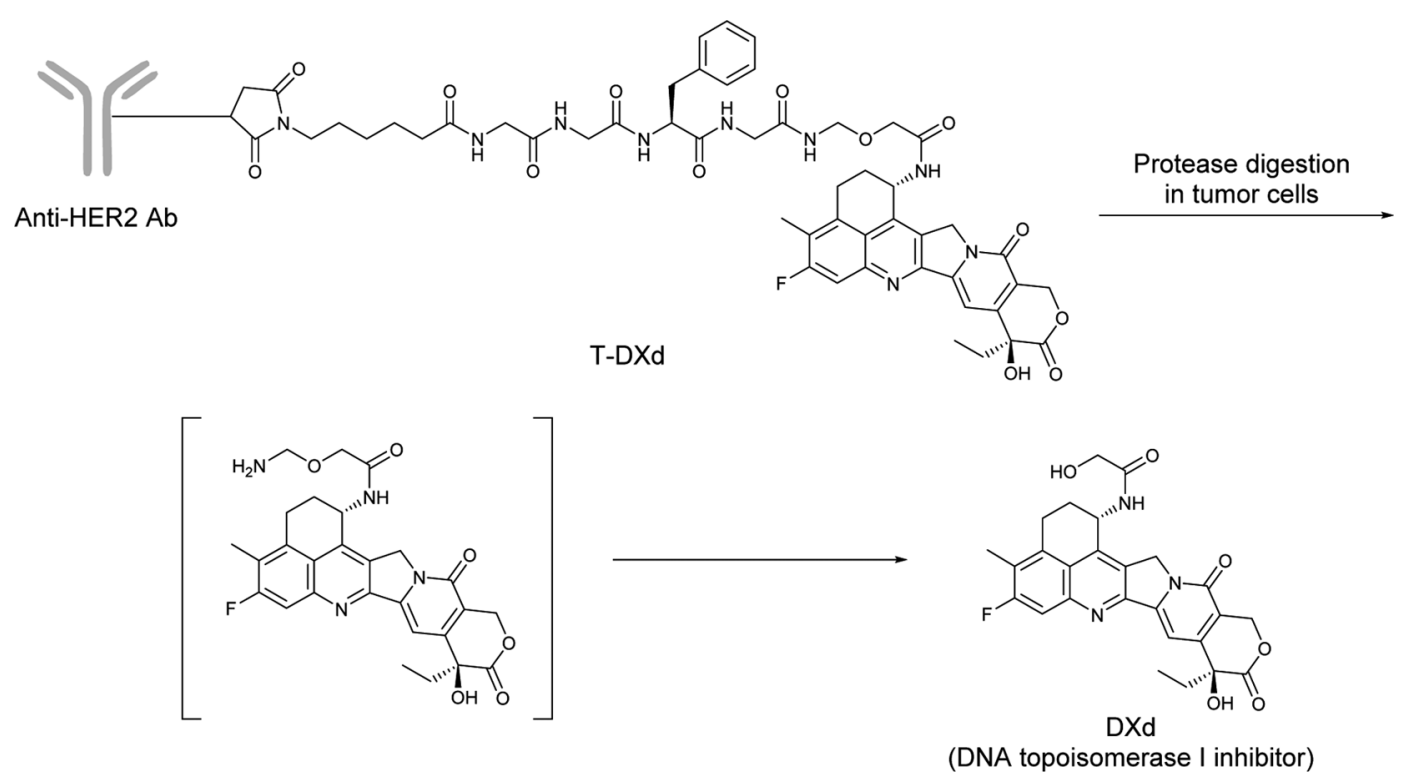

Fig. 3. Plausible mechanism of payload (DXd) release from trastuzumab deruxtecan (T-DXd) in tumor tissues. 
than T-DM1 or the ADC with DAR of 3.4, and that T-DXd exhibited preliminary evidence of activity in cancers with low HER2 expression.

\section{Non-clinical Study of Bystander Antitumor Effect}

DXd exerts antitumor activity through cytotoxicity not only on the target cells, but also on neighboring tumor cells, as a result of the membrane permeability of the payload (see
Fig. 5). This is known as the bystander antitumor effect of ADCs $[14,19,20]$.

The bystander antitumor efficacy of T-DXd was confirmed by a xenograft mouse model grafted with heterogeneous tumor tissues generated by inoculating a mixture of NCI-N87 (HER2-positive cell line) and MDA-MB-468-Luc (HER2negative and luciferase-expressing reporter cell line) [14]. HER2-targeting ADC, T-DXd or T-DM1, or control ADC was administered to the co-inoculated xenograft mice, and tumor volume and luciferase activity of each mouse were

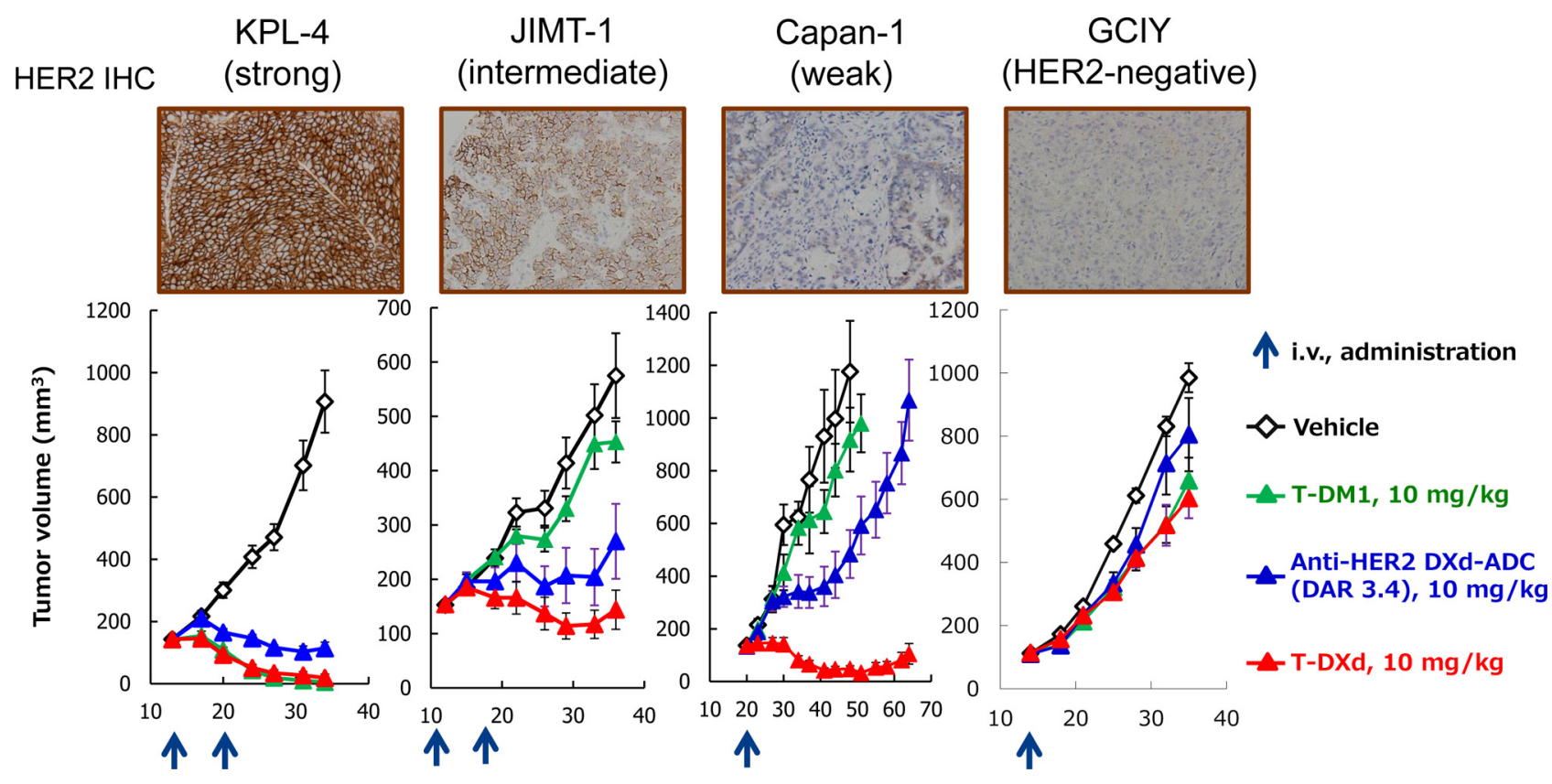

Fig. 4. Antitumor activity of trastuzumab deruxtecan (T-DXd) was evaluated in mouse xenograft models in comparison with trastuzumab emtansine (T-DM1) and anti-HER2 DXd- antibody-drug conjugate (ADC) (DAR 3.4). The stained images in each collected tumor were assessed by HER2 immunohistochemistry (IHC) using Dako HercepTest II kit (Dako, Glostrup, Denmark). KPL-4 (strong stain) and JIMT-1 (intermediate stain) showed HER2 positivity, Capan-1 (weak stain) showed low HER2 expression, and GCIY showed HER2 negativity. Arrows show the days of intravenous administration of each ADC. ADC was dosed once or twice every other week.

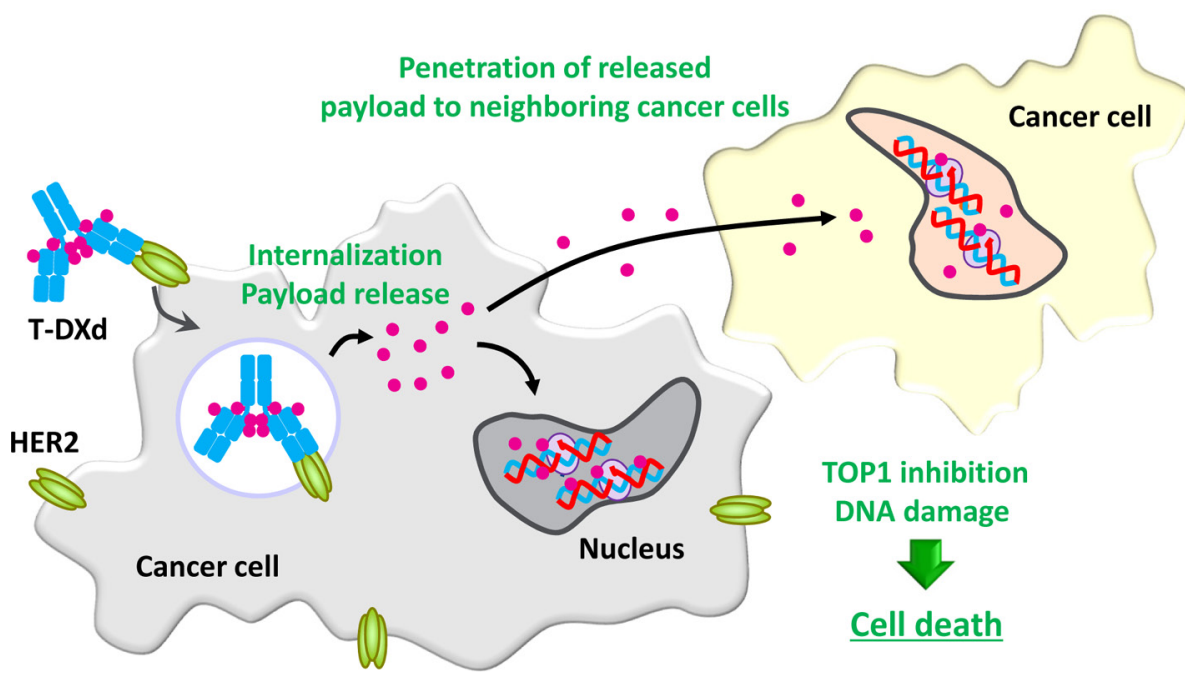

Fig. 5. Bystander antitumor effect of trastuzumab derxtecan (T-DXd). 

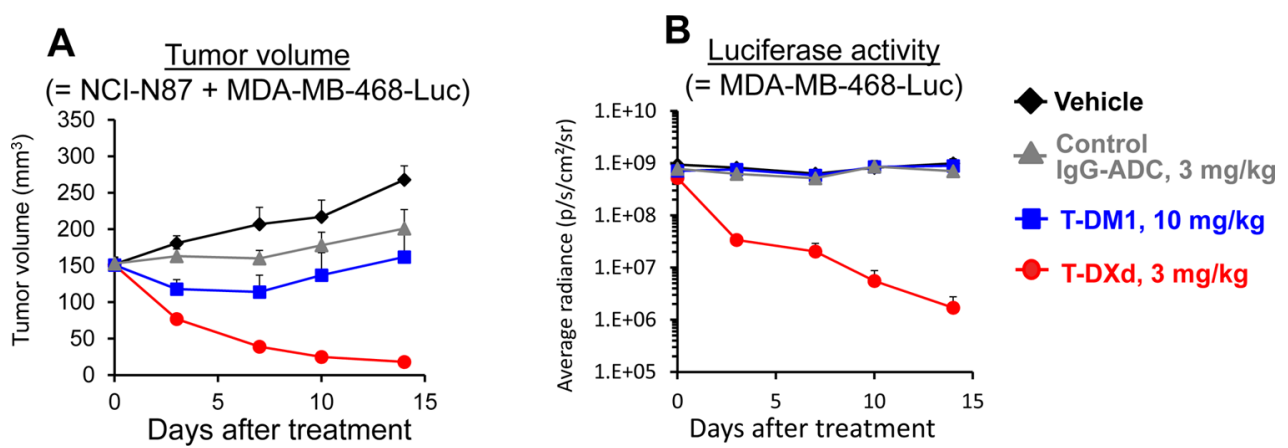

Fig. 6. Antitumor activity of antibody-drug conjugates against co-inoculation of HER2-positive and HER2-negative tumor cells. A: Tumor volume was confirmed antitumor activity against both HER2-positive and -negative tumor cells. B: Luciferase activity was detected by in vivo imager after administration of substrate.

measured, as shown in Fig. 6. In the T-DXd-treated group, an apparent reduction of luciferase signal was observed, indicating that MDA-MB-468-Luc cells were completely eradicated by T-DXd. Similar results were not observed with T-DM1 or control ADC. In terms of changes in tumor volume, T-DM1 inhibited tumor growth but was less potent than T-DXd, which could be attributed to the limited with only the HER2-positive cells being eliminated. In the TDM1-treated group, immunohistochemical analysis of the treated tumors showed the eradication of HER2-positive cells, and HER2-negative cancer cells occupied a large part of the tumor tissue. In the T-DXd-treated group, almost all of the HER2-positive and HER-negative cells disappeared and there were few or no cancer cells remaining in the tumors. These results clearly demonstrate that T-DXd showed antitumor activity against not only HER2-positive tumor cells but also HER2-negative cells under the co-inoculated conditions, whereas T-DM1 did not affect HER2-negative tumor cells.

These results suggest that T-DXd is effective even in tumors with low HER2 expression levels, as it can efficiently deliver the cytotoxic payload as a result of the high DAR and high linker stability and be effective in a heterogeneous tumor environment as a result of the bystander antitumor effect.

\section{Clinical Trials of Trastuzumab Deruxtecan}

In non-clinical studies, T-DXd inhibited the growth of both high- and low-HER2-expressing tumors, including those resistant to T-DM1. In a phase 1 (NCT02564900) dose-escalation study carried out to investigate the safety and tolerability of T-DXd [21], 24 patients received initial intravenous doses of T-DXd of between 0.8 and $8.0 \mathrm{mg} /$ $\mathrm{kg}$, with dose-limiting toxicity (DLT) assessed on a 21-day cycle. DLT was not observed and a single-dose maximum tolerated dose (MTD) was not reached even at $8.0 \mathrm{mg} / \mathrm{kg}$, which is the highest dose of trastuzumab used clinically.

The DESTINY-Breast01 pivotal study (phase 2 consisting of two parts; an open-label, single-group study, NCT03248492) evaluated T-DXd in adults with pathologically documented HER2-positive metastatic breast cancer who had received previous treatment with T-DM1 [22, 23].
A total of 184 patients who had undergone a median of six previous treatments received the recommended dose of T-DXd (5.4 mg/kg, once every 3 weeks). In the intentionto-treat analysis (cut-off date of August 1, 2019), responses to therapy were reported in 112 patients [overall response rate (ORR) $60.9 \%$, 95\% confidence interval (CI), 53.4 to 68.0 ] and the median progression-free survival (PFS) was 16.4 months (95\% CI, 12.7 to not reached). During the study, the most common adverse events of grade 3 or higher were a decreased neutrophil count (in $20.7 \%$ of the patients), anemia (in 8.7\%), and nausea (in 7.6\%). On independent adjudication, T-DXd treatment was associated with interstitial lung disease (ILD) in $13.6 \%$ of the patients (grade 1 or $2,10.9 \%$; grade 3 or $4,0.5 \%$; and grade $5,2.2 \%$ ).

These results showed that T-DXd exhibited durable antitumor activity in a pretreated patient population with HER2-positive metastatic breast cancer. In addition to gastrointestinal toxicity and myelosuppression, ILD was observed in a subgroup of patients, which requires attention to pulmonary symptoms and close monitoring. Elucidation of the mechanism behind the onset of ILD is extremely important for the prediction and treatment of this disease. Understanding this mechanism is the goal of further research for ADC therapy.

\section{Conclusions}

The optimized linker structure of T-DXd led to the maximum number of drugs conjugated to an antibody by overcoming the hydrophobicity of the payload drug without compromising the physicochemical properties of the antibody. In non-clinical study, T-DXd, which utilizes this optimized linker-payload system, was shown to exhibit outstanding antitumor efficacy by efficiently releasing its payload in the target tumor tissue while remaining stable in systemic circulation [11]. In addition, the payload released from T-DXd, the DXd moiety, is a potent TOP1 inhibitor. DXd retained high TOP1 inhibition as supported by studies of the structure-activity relationship of the molecule. Furthermore, DXd was able to impart a bystander antitumor effect, potently attacking neighboring tumor cells including target-negative tumor cells. Thus, it is expected to be active in heterogeneous tumors because it has membrane 
permeability. T-DXd showed significant antitumor efficacy against many HER2-positive and HER2-low-expression tumor models including T-DM1-resistant ones. A first-inhuman phase 1 study of T-DXd was initiated in September 2015 , followed by a pivotal phase 2 ("DESTINY") study. T-DXd was approved for the treatment of patients with HER2-positive unresectable or recurrent breast cancer by the US FDA in December 2019, the Japanese Ministry of Health, Labour and Welfare (MHLW) in March 2020, the European Medicines Agency in January 2021, and Health Canada's Notice and UK's National Institute for Health and Care Excellence in April 2021.

Additionally, T-DXd was approved for the treatment of patients with HER2-positive unresectable or recurrent gastric cancer by MHLW in September 2020 and by the US FDA in January 2021 [24, 25]. Many other clinical trials are now ongoing for the treatment of multiple cancer types, with therapeutic efficacy having tentatively been confirmed for HER2-expressing or HER2-mutant solid tumors, including breast cancer, gastric cancer, non-small cell lung cancer, colorectal cancer.

The outcomes suggest that T-DXd incorporating the novel optimized linker is a potent, new class of anti-HER2 ADC, which can provide clinical benefit to many patients with HER2-expressing and HER2-mutant cancers.

\section{Conflict of Interest}

The author is an employee of Daiichi Sankyo Co., Ltd.

\section{References}

1.Lambert, J. M. and Morris, C. Q. 2017. Antibody-drug conjugates (ADCs) for personalized treatment of solid tumors: A review. Adv. Ther. 34: 1015-1035. [Medline] [CrossRef]

2.Chari, R. V. J., Miller, M. L. and Widdison, W. C. 2014. Antibody-drug conjugates: an emerging concept in cancer therapy. Angew. Chem. Int. Ed. Engl. 53: 3796-3827. [Medline] [CrossRef]

3.Ducry, L. and Stump, B. 2010. Antibody-drug conjugates: linking cytotoxic payloads to monoclonal antibodies. Bioconjug. Chem. 21: 5-13. [Medline] [CrossRef]

4.Panowski, S., Bhakta, S., Raab, H., Polakis, P. and Junutula, J. R. 2014. Site-specific antibody drug conjugates for cancer therapy. MAbs 6: 34-45. [Medline] [CrossRef]

5.Agarwal, P. and Bertozzi, C. R. 2015. Site-specific antibody-drug conjugates: the nexus of bioorthogonal chemistry, protein engineering, and drug development. Bioconjug. Chem. 26: 176-192. [Medline] [CrossRef]

6.Perez, H. L., Cardarelli, P. M., Deshpande, S., Gangwar, S., Schroeder, G. M., Vite, G. D. and Borzilleri, R. M. 2014. Antibody-drug conjugates: current status and future directions. Drug Discov. Today 19: 869-881. [Medline] [CrossRef]

7.Feld, J., Barta, S. K., Schinke, C., Braunschweig, I., Zhou, Y. and Verma, A. K. 2013. Linked-in: design and efficacy of antibody drug conjugates in oncology. Oncotarget 4: 397-412. [Medline] [CrossRef]

8.Joubert, N., Beck, A., Dumontet, C. and Denevault-Sabourin, C. 2020. Antibody-drug conjugates: the last decade. Pharmaceuticals (Basel) 13: 245. [Medline] [CrossRef]

9.Nakada, T., Sugihara, K., Jikoh, T., Abe, Y. and Agatsuma, T. 2019. The latest research and development into the antibodydrug for HER2 cancer therapy. Chem. Pharm. Bull. (Tokyo) 67:
173-185. [Medline] [CrossRef]

10. Ogitani, Y., Aida, T., Hagihara, K., Yamaguchi, J., Ishii, C., Harada, N., Soma, M., Okamoto, H., Oitate, M., Arakawa, S., Hirai, T., Atsumi, R., Nakada, T., Hayakawa, I., Abe, Y. and Agatsuma, T. 2016. DS-8201a, a novel HER2-targeting ADC with a novel DNA topoisomerase I inhibitor, demonstrates a promising antitumor efficacy with differentiation from T-DM1. Clin. Cancer Res. 22: 5097-5108. [Medline] [CrossRef]

11. Okamoto, H., Oitate, M., Hagihara, K., Shiozawa, H., Furuta, Y., Ogitani, Y. and Kuga, H. 2020. Pharmacokinetics of trastuzumab deruxtecan (T-DXd), a novel anti-HER2 antibody-drug conjugate, in HER2-positive tumour-bearing mice. Xenobiotica 50: 1242-1250. [Medline] [CrossRef]

12. Nagai, Y., Oitate, M., Shiozawa, H. and Ando, O. 2019. Comprehensive preclinical pharmacokinetic evaluations of trastuzumab deruxtecan (DS-8201a), a HER2-targeting antibody-drug conjugate, in cynomolgus monkeys. Xenobiotica 49: 1086-1096. [Medline] [CrossRef]

13. Shiose, Y., Kuga, H., Ohki, H., Ikeda, M., Yamashita, F. and Hashida, M. 2009. Systematic research of peptide spacers controlling drug release from macromolecular prodrug system, carboxymethyldextran polyalcohol-peptide-drug conjugates. Bioconjug. Chem. 20: 60-70. [Medline] [CrossRef]

14. Ogitani, Y., Hagihara, K., Oitate, M., Naito, H. and Agatsuma, T. 2016. Bystander killing effect of DS-8201a, a novel anti-human epidermal growth factor receptor 2 antibodydrug conjugate, in tumors with human epidermal growth factor receptor 2 heterogeneity. Cancer Sci. 107: 1039-1046. [Medline] [CrossRef]

15. Yan, M., Parker, B. A., Schwab, R. and Kurzrock, R. 2014. HER2 aberrations in cancer: implications for therapy. Cancer Treat. Rev. 40: 770-780. [Medline] [CrossRef]

16. Yan, M., Schwaederle, M., Arguello, D., Millis, S. Z., Gatalica, Z. and Kurzrock, R. 2015. HER2 expression status in diverse cancers: review of results from 37,992 patients. Cancer Metastasis Rev. 34: 157-164. [Medline] [CrossRef]

17. Goldhirsch, A., Winer, E. P., Coates, A. S., Gelber, R. D., Piccart-Gebhart, M., Thürlimann, B., Senn H. J., Panel members. 2013. Personalizing the treatment of women with early breast cancer: highlights of the St Gallen International Expert Consensus on the Primary Therapy of Early Breast Cancer 2013. Ann. Oncol. 24: 2206-2223. [Medline] [CrossRef]

18. Schalper, K. A., Kumar, S., Hui, P., Rimm, D. L. and Gershkovich, P. 2014. A retrospective populationbased comparison of HER2 immunohistochemistry and fluorescence in situ hybridization in breast carcinomas: impact of 2007 American Society of Clinical Oncology/ College of American Pathologists criteria. Arch. Pathol. Lab. Med. 138: 213-219. [Medline] [CrossRef]

19. Polakis, P. 2016. Antibody drug conjugates for cancer therapy. Pharmacol. Rev. 68: 3-19. [Medline] [CrossRef]

20. Staudacher, A. H. and Brown, M. P. 2017. Antibody drug conjugates and bystander killing: is antigen-dependent internalisation required? Br. J. Cancer 117: 1736-1742. [Medline] [CrossRef]

21. Doi, T., Shitara, K., Naito, Y., Shimomura, A., Fujiwara, Y., Yonemori, K., Shimizu, C., Shimoi, T., Kuboki, Y., Matsubara, N., Kitano, A., Jikoh, T., Lee, C., Fujisaki, Y., Ogitani, Y., Yver, A. and Tamura, K. 2017. Safety, pharmacokinetics, and antitumour activity of trastuzumab deruxtecan (DS-8201), a HER2-targeting antibody-drug conjugate, in patients with advanced breast and gastric or gastro-oesophageal tumours: a phase 1 dose-escalation study. Lancet Oncol. 18: 1512-1522. [Medline] [CrossRef]

22. Tamura, K., Tsurutani, J., Takahashi, S., Iwata, H., Krop, I. E., Redfern, C., Sagara, Y., Doi, T., Park, H., Murthy, R. K., Redman, R. A., Jikoh, T., Lee, C., Sugihara, M., Shahidi, J., Yver, A. and Modi, S. 2019. Trastuzumab deruxtecan (DS8201a) in patients with advanced HER2-positive breast 
cancer previously treated with trastuzumab emtansine: a dose-expansion, phase 1 study. Lancet Oncol. 20: 816-826. [Medline] [CrossRef]

23. Modi, S., Saura, C., Yamashita, T., Park, Y. H., Kim, S.B., Tamura, K., Andre, F. and Iwata, H. Y., Ito, Y., Tsurutani, J., Sohn, J., Denduluri, N., Perrin, C., Aogi, K., Tokunaga, E., Im, S.-A., Lee, K. S., Hurvitz, S. A., Cortes, J., Lee, C., Chen, S., Zhang, L., Shahidi, J., Yver, A. and Krop, I. 2020. Trastuzumab deruxtecan in previously treated HER2positive breast cancer. N. Engl. J. Med. 382: 610-621.

24. Shitara, K., Iwata, H., Takahashi, S., Tamura, K., Park, H., Modi, S., Tsurutani, J., Kadowaki, S., Yamaguchi, K., Iwasa,
S., Saito, K., Fujisaki, Y., Sugihara, M., Shahidi, J. and Doi, T. 2019. Trastuzumab deruxtecan (DS-8201a) in patients with advanced HER2-positive gastric cancer: a dose-expansion, phase 1 study. Lancet Oncol. 20: 827-836. [Medline] [CrossRef] 25. Shitara, K., Bang, Y. J., Iwasa, S., Sugimoto, N., Ryu, M. H., Sakai, D., Chung, H. C., Kawakami, H., Yabusaki, H., Lee, J., Saito, K., Kawaguchi, Y., Kamio, T., Kojima, A., Sugihara, M., Yamaguchi K., DESTINY-Gastric01 Investigators. 2020. Trastuzumab deruxtecan in previously treated HER2positive gastric cancer. N. Engl. J. Med. 382: 2419-2430. [Medline] [CrossRef] 